\title{
Induction of UDP-glucuronosyltransferase 1A by naturally occurring phytochemicals in human hepatoma cells
}

\author{
Violetta Krajka-Kuźniak, Adam Krysztofiak, Jarosław Paluszczak \\ Department of Pharmaceutical Biochemistry, Poznan University of Medical Sciences, Poland
}

\begin{abstract}
UDP-glucuronosyltransferases (UGTs) are important detoxification and drug-metabolizing enzymes, which catalyse the glucuronidation of exogenous and endogenous chemicals. The anti-carcinogenic activity of dietary phytochemicals is partly attributed to the induction of phase II enzymes, including UGT1A. Our earlier study showed that protocatechuic acid increased UGT activity in rat liver. A similar effect was observed for indole-3carbinol and phenethyl isothiocyanate in rat liver. In this study we assessed the effect of protocatechuic acid, tannic acid, indole-3-carbinol and phenethyl isothiocyanate on the expression and protein level of UGT1A in hepatocellular carcinoma HepG2 cells. Cells were incubated with $2 \mu \mathrm{M}$ and $10 \mu \mathrm{M}$ of protocatechuic acid, tannic acid, or indole-3-carbinol and $1 \mu \mathrm{M}$ and $5 \mu \mathrm{M}$ of phenethyl isothiocyanate for 72 hours. Transcript level was measured by RT-PCR and protein level by the immunoblot assay. Treatment with protocatechuic acid, tannic acid, indole-3-carbinol and phenethyl isothiocyanate induced the expression and protein level of UGT1A. Phenethyl isothiocyanate increased the mRNA of UGT1A to the most extent $(0.28-5.7$ fold change, $p<0.05)$. Increased expression of UGT1A was accompanied by the enhancement of its protein level, with the exception of protocatechuic acid at the dose of $2 \mu \mathrm{M}$. Overall, isothiocyanates and indoles were more potent as UGT inducers than phenolic acids. Collectively, the results suggest that the induction of UGT1A could contribute to the hepatoprotective and chemopreventive properties of these phytochemicals.
\end{abstract}

Keywords: tannic acid, protocatechuic acid, indole-3-carbinol, phenethyl isothiocyanate, UGT1A, HepG2.

\section{Introduction}

Uridine diphosphoglucuronosyltransferases (UGTs) are phase II enzymes that conjugate metabolic intermediates with glucuronic acid to form glucuronides, which are characterized by increased solubility in water and therefore are more easily excreted from the body [1]. Two general classes of UGTs exist in humans: UGT1 and UGT2. Both classes contain multiple members that catalyze the glucuronidation of a diverse array of substrates and are differentially expressed in various organs [2]. UGT1A enzymes metabolize both endogenous and exogenous compounds such as bilirubin and drugs, whereas UGT2B enzymes target rather endogenous compounds such as steroid hormones [3].
Several studies demonstrated that diets containing plant foods, particularly cruciferous vegetables induce drug metabolizing enzymes in humans and rodents [4-7]. Glucosinolates present in Cruciferae vegetables are hydrolyzed by myrosinase during food processing to biologically active indoles and isothiocyanates. The structure of these phytochemicals is shown in Figure 1. In 1997, Taioli et al. [8] reported the induction of UGT activity in humans fed $400 \mathrm{mg}$ of indole-3-carbinol for 5 days. Other studies showed that indole-3-carbinol increased UGT activity in male Wistar rats [9]. Phenethyl isothiocyanate increased hepatic UGT activity in male Fisher 344 rats [10]. A similar effect has been observed in the case of phenolic acids, which are prev- 
alent among many edible plants. Protocatechuic acid, a simple phenolic acid (Figure 1) increased UGT activity in the epidermis of female Swiss mice [11]. On the other hand, tannic acid, which is a mixture of digallic acid esters of glucose, did not significantly change hepatic activity of UGT in Wistar rats [12]. These studies indicate that phytochemicals may increase the activity of UGT in in vivo models. Thus, the up-regulation of this enzyme by phenolic acids, indoles and isothiocyanates may also represent one of the cancer preventive mechanisms of these compounds.

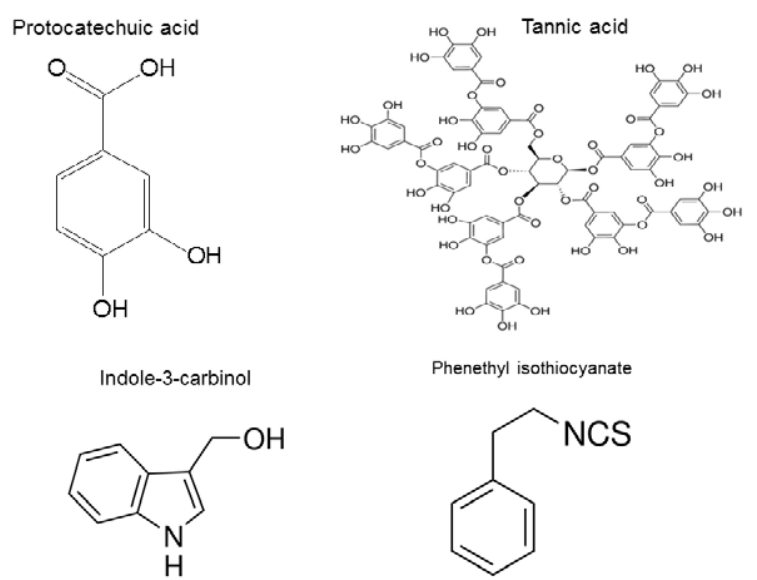

Figure 1. Chemical structure of protocatechuic acid, tannic acid, indole3-carbinol and phenethyl isothiocyanate

Liver is the major organ for glucuronidation of a variety of xenobiotics. Carcinogens, including benzo(a) pyrene, are conjugated by UGT1A, and increased glucuronidation would result in their increased elimination and potentially decreased toxicity [13]. In this regard, the effective neutralization of chemical mutagens may significantly reduce the risk of cancer initiating events.

Therefore, the objective of this study was to evaluate the effects of phytochemicals such as tannic acid, protocatechuic acid, indole-3-carbinol and phenethyl isothiocyanate on the expression of UGT in human hepatocellular carcinoma HepG2 cells.

\section{Material and methods}

Chemicals and antibodies

Antibiotics solution $\left(10^{4} \mathrm{U}\right.$ penicillin, $10 \mathrm{mg}$ streptomycin, $25 \mu \mathrm{g}$ amphotericin B), dimethyl sulfoxide (DMSO), fetal bovine serum (FBS), Dulbecco's Modified Eagle's Medium (DMEM), Radio-Immunoprecipitation Assay (RIPA) buffer, Tris were obtained from Sigma-Aldrich (St. Louis, MO, USA). HepG2 (ATCC HB 8065) cells were provided by Prof. Zofia Mazerska from the Department of Pharmaceutical Technology and Biochemistry, Gdańsk University of Technology, Poland. All the primers used in PCR reactions were obtained from oligo. pl (Warsaw, Poland). Protease inhibitor cocktail was obtained from Roche Diagnostics $\mathrm{GmbH}$ (Penzberg, Germany). Primary and secondary antibodies against UGT and $\beta$-actin were supplied by Santa Cruz Biotechnology (Santa Cruz, CA, USA). Rainbow colored protein molecular weight marker was purchased from Amersham Pharmacia Biotechnology (Piscataway, NJ, USA). All the other chemicals were commercial products of the highest purity available.

\section{Cell culture and treatment}

HepG2 cells were maintained in DMEM containing $10 \%$ fetal bovine serum and antibiotics. The cells were grown in a humidified incubator at $37^{\circ} \mathrm{C}$ in the atmosphere of $5 \% \mathrm{CO}_{2}$. To assess the effect of tannic acid, protocatechuic acid, indole-3-carbinol and phenethyl isothiocyanate on the measured parameters, $5 \times 10^{5}$ cells were seeded per $100 \mathrm{~mm}$ culture dish. After 24 hours of initial incubation the cells were treated with $2 \mu \mathrm{M}$ and $10 \mu \mathrm{M}$ tannic acid, protocatechuic acid or indole-3-carbinol and $1 \mu \mathrm{M}$ and $5 \mu \mathrm{M}$ phenethyl isothiocyanate or $0.1 \%$ vehicle control. The incubation was continued for subsequent 72 hours and cells were harvested.

RNA isolation and total protein lysates preparation Extraction of total RNA from cells was performed using GeneMatrix Universal DNA/RNA/Protein Purification Kit (EurX, Poland) according to the manufacturer's instructions. Whole cell lysates were prepared using RIPA buffer.

\section{Quantitative PCR}

Total RNA was subjected to reverse transcription using RevertAid Kit (Fermentas, Burlington, Canada) followed by quantitative real-time PCR. For real-time analyses the Maxima SYBR Green Kit (Fermentas) and a BioRad Chromo4 thermal cycler were used. The protocol started with 5 minutes enzyme activation at $95^{\circ} \mathrm{C}$, followed by 40 cycles of $95^{\circ} \mathrm{C}$ for $15 \mathrm{~s}, 54^{\circ} \mathrm{C}$ for $20 \mathrm{~s}$ and $72^{\circ} \mathrm{C}$ for $40 \mathrm{~s}$ and final elongation at $72^{\circ} \mathrm{C}$ for 5 minutes. Melting curve analysis was used for product verification. The estimation of the expression of TBP (TATA box binding protein) and PBGD (porphobilinogen deaminase) was used for data normalization. Primer sequences for UGT1A transcript analysis were as follows: 5'-AACGATCTGCTTGGTCAC-3' (forward) and 5'-GAACATTCAGGGTCACTCC-3' (reverse). 


\section{Western blot analysis}

For the determination of the level of UGT protein the immunoblot assay was used. Protein content in the samples was determined by the Lowry method [14]. All the experiments were repeated three times. Whole cell lysate samples containing $100 \mu \mathrm{g}$ proteins were separated in 10\% SDS-PAGE slab gels and proteins were transferred to nitrocellulose membranes. After blocking with $10 \%$ skimmed milk, proteins were probed with goat polyclonal UGT and/or rabbit polyclonal $\beta$-actin antibodies. Estimation of the level of $\beta$-actin was used as an internal control. As the secondary antibodies in the staining reaction, the peroxidase-labeled anti-goat $\lg \mathrm{G}$ or anti-rabbit $\lg \mathrm{G}$ were used. Bands were visualized with Advansta Western Bright Quantum Western blotting detection kit. The amount of the immunoreactive product in each lane was determined using the Quantity One software (BioRad Laboratories, Hercules, CA, USA). Values were calculated as relative absorbance units (RQ) per mg protein.

\section{Statistical analysis}

The statistical analysis was performed by one-way ANOVA. The statistical significance between the experimental groups and their respective controls was assessed by Tukey's post hoc test, with $p<0.05$.

\section{Results}

\section{Analysis of the expression of UGT1A}

The expression of UGT1A was measured by quantitative PCR (Figure 2). The expression of UGT1A mRNA increased after treatment of $\mathrm{HepG} 2$ cells with all the tested compounds. UGT1A mRNA level was enhanced by (0.28-5.7-fold) after treatment with either dose of phenethyl isothiocyanate. Indole-3-carbinol in either dose induced about 1.14-1.83-fold higher expression of UGT1A. Tannic acid in either dose increased about 0.48-0.96-fold the expression of UGT1A, while protocatechuic acid increased about 0.27 -fold the expression of UGT only in the higher dose.

\section{Evaluation of the protein level of UGT1A}

The level of UGT1A protein in HepG2 cells was investigated using the Western blot assay with a specific antibody against the enzyme (Figure 3 ). The content of UGT1A (Figure 3) increased by about 28-75\% after the treatment with either phenethyl isothiocyanate or indole-3-carbinol in HepG2 cells. The level of UGT1A increased by $26-45 \%$ after the treatment with 2 and $10 \mu \mathrm{M}$ tannic acid and $10 \mu \mathrm{M}$ protocatechuic acid in HepG2 cells. No statistically significant changes in the content of the analyzed protein were detected in HepG2 cells as the result of treatment with the protocatechuic acid at the dose of $2 \mu \mathrm{M}$ (Figure 3).

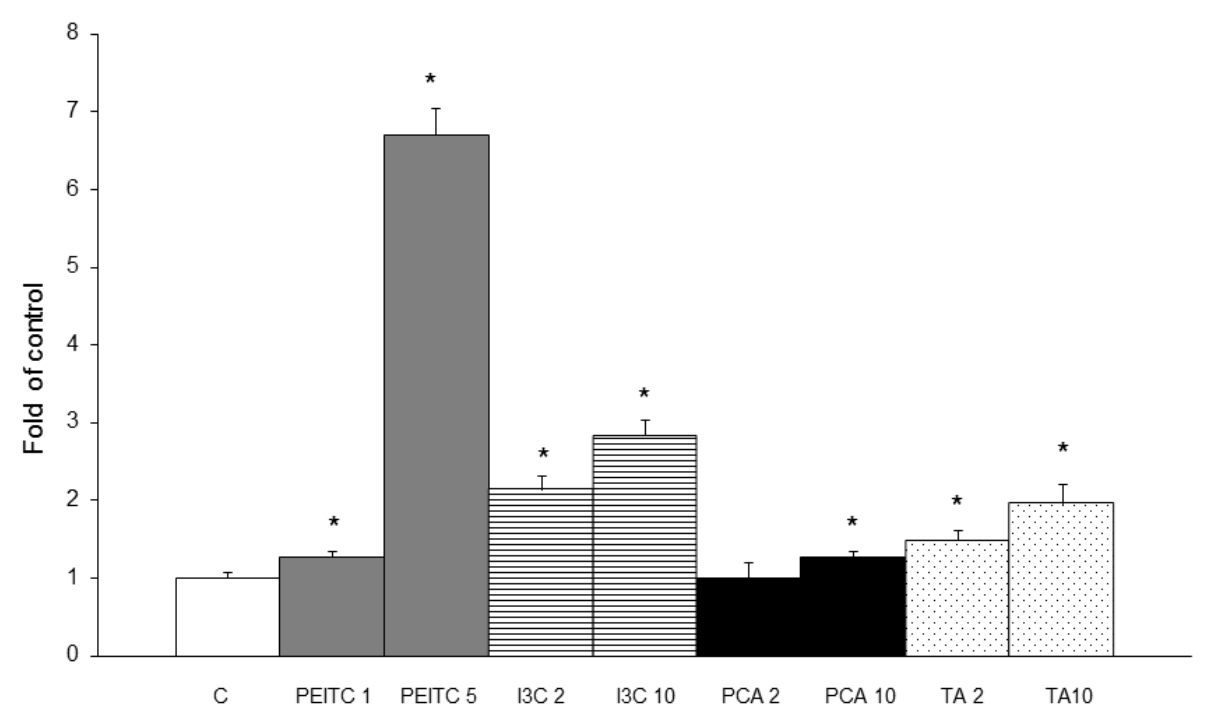

Figure 2. The effect of phenethyl isothiocyanate, indole-3-carbinol, protocatechuic acid and tannic acid on UGT1A transcript level in HepG2 cells. Data (mean \pm SEM) from three separate experiments are presented, the asterisk above the bar denotes statistically significant differences from the control group, $p<0.05$

C: vehicle control; PEITC: phenethyl isothiocyanate; IBC: indole-3-carbinol; PCA: protocatechuic acid; TA: tannic acid 
A)

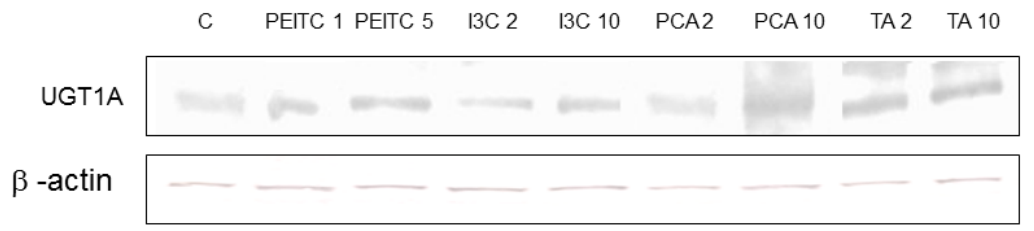

B)

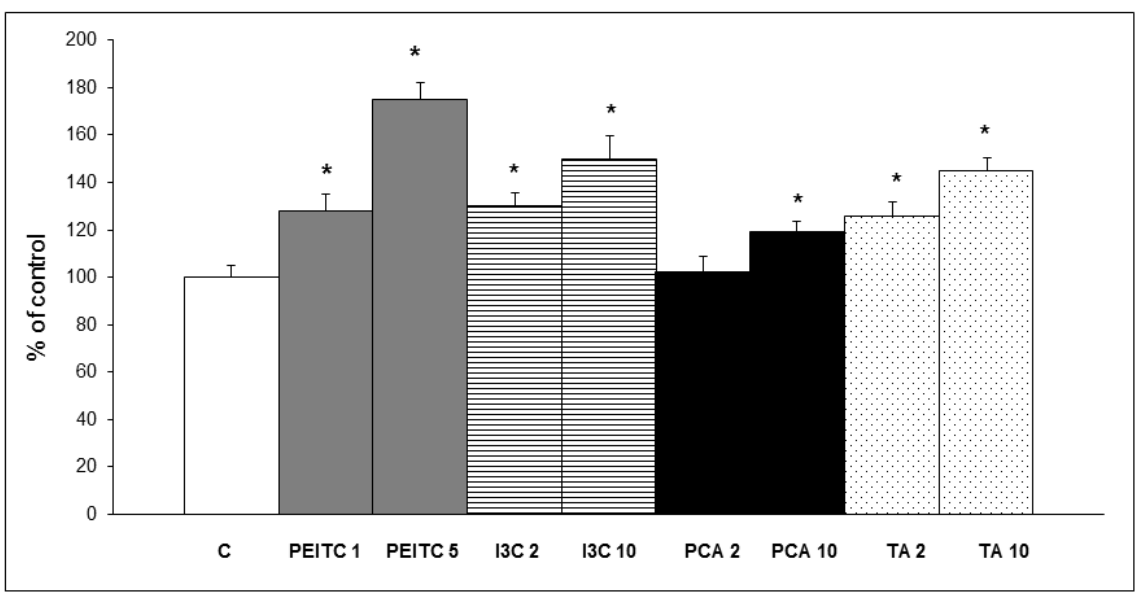

Figure 3. The effect of phenethyl isothiocyanate, indole-3-carbinol, protocatechuic acid and tannic acid on the level of the UGT1A protein in HepG2 cells. (A) Representative immunoblots for the analysis of UGT1A protein; $\beta$-actin was used as a normalization control. (B) Results of Western blot analysis of the content of UGT1A protein in comparison to the control group. Data (mean \pm SEM) from three separate experiments are presented, the asterisk above the bar denotes statistically significant differences from the control group, $p<0.05$

\section{Discussion}

It is well known that dietary factors affect the development of human cancers. The human diet contains a large number of a variety of compounds that may inhibit mutagenesis and/or carcinogenesis [15-18]. These compounds are very diverse in chemical structures and their protective mechanisms are generally unclear. Although the prevention of cancer may be due to multiple mechanisms, one mode of action of anti-carcinogens may be to enhance the carcinogen detoxification systems, such as UGT [19]. UGT can minimize carcinogenicity by conjugation of chemicals with glucuronic acid, which in general results in the generation of biologically less active molecules and increases water-solubility of the conjugated products, which facilitates their excretion from the body via bile or urine [20]. Therefore, the enhancement of the activity of UGT could potentially augment the capacity to withstand the burden of toxic agents and (pre)carcinogens to wchich humans are exposed daily [21]. In this regard, it was shown that UGT1A-deficient rats show reduced capacity to glucuronidate benzo(a)pyrene, a mutagenic polycyclic aromatic hydrocarbon and are more susceptible to formation of DNA adducts [22, 23]. In addition, Berges et al. [24] reported that UGT activity was inversely correlated with the number of preneoplastic liver foci in rats, supporting a role of UGT induction in cancer chemoprevention.

Increased glucuronidation could mediate the protective and antioxidant effects of chemopreventive compounds. Several studies which were performed in animals models indicated that naturally occurring dietary anticarcinogens may be able to elevate UGT activity [25, 26]. In particular, isothiocyanates such as sulforaphane and phenethyl isothiocyanate, that exhibit chemopreventive properties have been shown to induce UGT in animal models $[27,28]$. In addition, whole tea, tea extracts, and polyphenols from different types of tea also induce UGT activity and/or expression in animals and cell culture systems [29]. Moreover, our previous study showed that simple phenolic acid, protocatechuic acid increased the activity of UGT in rat liver [30].

In this study, we evaluated the ability of different plant phenolic acids and glucosinolates to modulate the UGT1A expression and protein level in HepG2 hepatoma cell line. Both phenolic acids induced UGT1A transcript in HepG2 cells. Tannic acid had a stronger effect on UGT1A than a simple phenolic acid, protocatechuic acid. However, the most potent inducers of UGT1A 
were glucosinolates: indole-3-carbinol and phenethyl isothiocyanate. Western blot analysis confirmed that the accumulation of transcript was accompanied by an increase in UGT1A protein level.

Our study showed that phenethyl isothiocyanate induced the expression and protein level of UGT1A to the most extent. A similar effect was observed by Sertzer et al. [9] and van der Loght [12] in rat liver. In contrast to our results, van der Loght et al. [12] observed no effects on hepatic UGT activity after treatment with indole-3-carbinol. This discrepancy may be related to differences in doses and/or species-specific effects.

The inducers of xenobiotic metabolism enzymes can be divided into monofunctional and bifunctional modulators [31]. Isothiocyanates are considered as monofunctional modulators since they induce the activity of only phase II enzymes. In our previous studies we confirmed that phenethyl isothiocyanate was the most effective inducer of phase II enzymes such as NQO1 and GST in rat liver [32]. This study shows that isothiocyanates and indoles are more active as inducers of phase II enzymes than phenolic acids in human hepatoma cells. Collectively, the results suggest that the induction of UGT1A may be one of the factors contributing to the hepatoprotective and chemopreventive properties of phenethyl isothiocyanate, indole-3-carbinol and, to a lesser extent, tannic acid and protocatechuic acid. Further studies are necessary to establish the direct relevance of the studied compounds in providing health benefits for humans.

\section{Acknowledgements}

This work was supported by the Poznan University of Medical Sciences grant STN 502-05-03302404-50737.

\section{Conflicts of interest statement}

All the authors declare no conflicts of interest.

\section{References}

1. Saracino MR, Lampe JW. Phytochemical regulation of UP-glucuronosyltransferase: implications for cancer prevention. Nutr. Cancer. 2007 Dec;59(2):121-41.

2. Owens IS, Basu NK, Banerjee R. UP-glucuronosyltransferases: gene structures of UT1 and UT2 families. Methods Enzymol. 2005 Dec;400(2):1-22.

3. Tukey RH, Strassburg CP. Human UP-glucuronosyltransferases: metabolism, expression and disease. Ann Rev Pharmacol Toxicol. 2000 Apr;40:581-616.

4. Pantuck EJ, Hsiao KC, Loub WD, Wattenberg LW, Kuntzman R. Stimulatory effect of vegetables on intestinal drug metabolism in the rat. JPharmacol Exp Ther. 1976 Aug;198(2): 278-83.

5. Navarro SL, Peterson S, Chen C, Makar KW, Schwarz Y, King IB et al. Cruciferous vegetable feeding alters UT1A1 activity: diet- and genotype-dependent changes in serum bilirubin in a controlled feeding trial. Cancer Prev Res (Phila). 2009 Apr;2(4):345-52.

6. Higdon JV, Delage B, Williams DE, Dashwodd RH. Cruciferous vegetables and human cancer risk: epidemiologic evidence and mechanistic basis. Pharmacol Res. 2007 Mar;55(3):224-36.

7. Robbins MG, Hauder J, Somoza V, Eshelman BD, Barnes DM, Hanlon PR. Induction of detoxification enzymes by feeding unblanched Brussels sprouts containing active myrosinase to mice for 2 wk. JFood Sci. 2010 Aug 1;75(6):H190-9.

8. Taioli E, Garbers S, Bradlow HL, Carmella SG, Akerkar S, Hecht SS. Effects of indole-3-carbinol on the metabolism of 4-(methylnitrosoamino)-1-(3-pyridyl)-1-butanone in smokers. Cancer Epidemiol Biomarkers Prev. 1997 Jul;6(7):517-22.

9. Shertzer HG, Sainsbury M. Chemoprotective and hepatic enzyme induction properties of indole and indenoindole antioxidants in rats. Food Chem Toxicol. 1991 Jun;29(6):391-400.

10. Dingley KH, Ubick EA, Chiarappa-Zucca ML, Nowell S, Abel S, Ebeler SE, et.al. Effect of dietary constituents with chemopreventive potential on adduct formation of low dose of the heterocyclic amines PIP and IQ and phase II hepatic enzymes. Nutr Cancer. 2003;46(2):212-21.

11. Szaefer H, Cichocki M, Brauze D, Baer-Dubowska W. Alteration in phase I and II enzyme activities and polycyclic aromatic hydrocarbons-DA adduct formation by plant phenolics in mouse epidermis. Nutr Cancer. 2004;48(1):70-7.

12. der Loght EJ, Roelofs HJ, Nanengast FM, Peters WM. Induction of rat hepatic and intestinal UP-glucuronosyltransferases by naturally occurring dietary anticarcinogens. Carcinogenesis. 2003 Oct;24(10):1651-6.

13. Telang U, Morris ME. Effect of orally administered phenethyl isothiocyanate on hepatic gene expression in rats. Mol Nutr Food Res. 2010 Dec;54(12):1802-6.

14. Lowry OH, Rosenbrough NJ, Farr AL, Randall RJ. Protein measurement with the Folin phenol reagent. JBiol Chem. 1951 Nov;193(1):265-75.

15. Terry P, Terry JB, Wolk A. Fruit and vegetable consumption in the prevention of cancer: an update. JIntern Med. 2001 Oct;250(4):280-90.

16. Fattahi S, Zabihi E, Abedian Z, Pourbagher R, Motevalizadeh Ardekani A et al. Total Phenolic and Flavonoid Contents of Aqueous Extract of Stinging Nettle and In Vitro Antiproliferative Effect on Hela and BT-474 Cell Lines. Int JMol Cell Med. 2014 Spring;3(2):102-7.

17. Koushik A, Hunter DJ, Spiegelman D, Beeson WL, van den Brandt PA, Buring JE et al. Fruits, vegetables, and colon cancer risk in a pooled analysis of 14 cohort studies. JNatl Cancer Inst. 2007 Oct 3;99(19):1471-83.

18. Lam TK, Gallicchio L, Lindsley K, Shields M, Hammond $\mathrm{E}$, Tao XG et al. Cruciferous vegetable consumption and lung cancer risk: a systematic review. Cancer Epidemiol Biomarkers Prev. 2009 Jan;18(1):184-95. 
19. Lasker J, Rickert DE. Absorption and glucuronylation of diethylstilbestrol by the rat small intestine. Xenobiotica. 1978 Nov;8(11):665-72.

20. Peters WM, Nagengast FM, van Tongeren JM. Glutathione S-transferase, cytochrome P450 and uridine 5'-diphosphateglucuronosyltransferase in human small intestine and liver. Gastroenterology. 1989 Mar;96(3):783-9.

21. Steinmetz KA, Potter JD. Vegetables, fruit and cancer. II. Mechanisms. Cancer Causes Control. 1991 Nov;2(6):42742.

22. Hu Z, Wells PG. Modulation of benzo[a]pyrene bioactivation by glucuronidation in lymphocytes and hepatic microsomes from rats with a hereditary deficiency in bilirubin UP-glucuronosyltransferase. Toxicol Appl Pharmacol. 1994 Aug;127(2):306-13.

23. Hu Z, Wells PG. In vitro and in vivo biotransformation and covalent binding of benzo(a)pyrene in Gunn and RA rats with a genetic deficiency in bilirubin uridine diphosphate-glucuronosyltransferase. JPharmacol Exp Ther 1992 Oct;263(1):334-42.

24. Berges R, Siess MH, Arnault I, Auger J, Kahane R, Pinnert MF et al. Comparison of the chemopreventive efficacies of garlic powders with different alliin contents against aflatoxin B1 carcinogenicity in rats. Carcinogenesis. 2004 Oct;25(10):1953-9.

25. Bu-Abbas A, Clifford MN, loannides C, Walker R. Stimulation of rat hepatic UP-glucuronosyltransferase activity following treatment with green tea. Food Chem Toxicol. $1995 \mathrm{Jan} ; 33(1): 27-30$

26. Bu-Abbas A, Clifford MN, Walker R, loannides C. Contribution of caffeine and flavanols in the induction of hepatic phase II activities by green tea. Food Chem Toxicol. 1998 Aug;36(8):617-21.
27. Bianchini $F$, Vainio $H$. Isothiocyanates in cancer prevention. Drug Metab Rev. 2004 Oct;36(3-4):655-67.

28. Conaway CC, Yang YM, Chung FL. Isothiocyanates as cancer chemopreventive agents: their biological activities and metabolism in rodents and humans. Curr Drug Metab. 2002 Jun;3(3):233-55.

29. Xu M, Bailey AC, Hernaez JF, Taoka CR, Schut HA, Dashwood RH. Protection by green tea, black tea, and indole-3-carbinol against 2-amino-3-methylimidazo[4,5 -f]quinoline-induced DA adducts and colonic aberrant crypts in the F344 rat. Carcinogenesis. 1996 Jul;17(7):1429-34.

30. Krajka-Kuźniak V, Szaefer H, Baer-Dubowska W. Modulation of cytochrome P450 and phase II enzymes by protocatechuic acid in mouse liver and kidney. Toxicology. 2005 Dec;216(1):24-31.

31. Talalay P. Chemoprotection against cancer by induction of phase 2 enzymes. Biofactors 2000 Jan;12(1-4):5-11.

32. Krajka-Kuźniak V, Szaefer H, Bartoszek A, Baer-Dubowska W. Modulation of rat hepatic and kidney phase II enzymes by cabbage juices: comparison with the effects of indole-3-carbinol and phenethyl isothiocyanate. Br JNutr. 2011 Mar;105(6):816-26.

\section{Correspondence address:} Violetta Krajka-Kuźniak Department of Pharmaceutical Biochemistry, Poznan University of Medical Sciences 4 Święcickiego Str., 60-781 Poznan, Poland phone: +48 618546623 fax: +48618546620 email: vkrajka@ump.edu.pl 